\title{
IMPACTOS DEL ASEGURAMIENTO DE LA CALIDAD Y ACREDITACIÓN DE LA EDUCACIÓN SUPERIOR. CONSIDERACIONES Y PROPOSICIONES
}

\section{Introducción ${ }^{2}$}

"Latin America's contemporary emphasis on accreditation and evaluation is welcome, indeed necessary (...) In any event, is important to avoid excess faith and over-reliance on any new tool. No system of evaluation will light a magic route to enhanced knowledge output. Instead, various kinds of evaluation must be coupled with various other means to achieve such output".

(Levy, 2002) $)^{3}$

Después de más de diez años de desarrollo de procesos de aseguramiento de la calidad (AC) y, particularmente, de acreditación de la educación superior en Chile, la pregunta por sus impactos comienza a concitar creciente interés. La instalación de este tipo de procesos en el país fue el resultado de aproximaciones sucesivas, conducidas con amplia participación y liderazgo. Se trató de iniciativas que supieron recoger la experiencia de universidades y organizaciones pioneras en temas de evaluación e incorporar las lecciones internacionales sobre la materia, obteniendo en un periodo muy corto logros que fueron mucho más allá de los objetivos originalmente planteados (INQAAHE Review Panel, 2005).

2 El presente artículo recoge partes de una sección de un estudio nacional realizado por los autores en el contexto del Proyecto ALFA Nro. DCI-ALA 2008/42: "Aseguramiento de la Calidad: Políticas Públicas y Gestión Universitaria", conducido por el Centro Interuniversitario de Desarrollo. El informe completo se encuentra disponible en: http:// www.cinda.cl/proyecto_alfa/htm/documentos.htm. Agradecemos a CINDA su apoyo para la realización del estudio, así como su autorización para publicar partes del informe nacional final. Los contenidos del artículo, sus limitaciones y problemas, son de exclusiva responsabilidad de los autores.

3 "El actual énfasis de Latinoamérica por la evaluación y acreditación es bienvenido, de hecho, necesario (...) En cualquier caso, es importante evitar un exceso de confianza y dependencia respecto de cualquier nueva herramienta. Ningún sistema de evaluación iluminará una ruta mágica a mejores resultados en el conocimiento. En su lugar, varios tipos de evaluación deben combinarse con varios otros medios para lograr tales resultados". 
Los inicios de la acreditación en Chile y la configuración de un sistema de AC de la educación superior estuvieron acompañados de un intenso debate acerca de sus potencialidades y limitaciones. Durante el desarrollo de los procesos experimentales de acreditación éste se dio particularmente en el gobierno, las comunidades académicas, asociaciones profesionales y disciplinarias, y otros actores claves vinculados con la educación superior. Luego, el debate se expandió al ámbito político nacional, a propósito de los más de cuatro años de tramitación del proyecto de ley sobre AC, que tuvo cerca de 800 indicaciones. En este proyecto la discusión se intensificó y polarizó, formulándose ciertos dilemas y tensiones que parecían tener relación más que con la promoción de la calidad, con profundas diferencias entre actores políticos sobre la función del Estado en la educación superior y ciertas visiones antagónicas cargadas de contenido ideológico (Armanet, 2005).

Años atrás, mientras algunos sostenían que los procesos de AC tenían el potencial de generar transformaciones importantes en nuestra educación superior, otros más bien planteaban que la acreditación, así como había sido propuesta, constituía una política centralizadora inútil o bien que terminaría por corroer la autonomía universitaria, capacidad de innovación y sana diversidad del sistema (Libertad y Desarrollo, 2003a y 2003b). Las perspectivas planteadas sobre los propósitos del AC y de la acreditación diferían ampliamente en el debate; mientras algunos querían que la acreditación apuntara fundamentalmente a regular la oferta (cual superintendencia de educación superior), otros sostenían que debía operar esencialmente como motor de mejoramiento, y otros que debía limitarse a entregar más y mejor información.

Entenderemos por AC a un conjunto de políticas, procedimientos, normas y prácticas (internas y externas a las instituciones y sus programas) destinadas a la mantención y promoción de estándares de calidad, así como a su divulgación y transparencia en el sistema. Por su parte, entenderemos por acreditación un modo particular de AC, que privilegia procedimientos de evaluación permanentes -internos y externos-, asociados con verificar y certificar públicamente que se cumplen ciertos criterios de calidad. Finalmente, existen diversas 
aproximaciones al concepto de "impacto", las que -cuál más cuál menos- se refieren a los cambios que razonablemente se pueden atribuir al AC y a la acreditación.

En el presente artículo nos interesa fundamentalmente una revisión de la literatura especializada y de la evidencia internacional sobre los impactos del AC, buscando en ellas ciertas claves que puedan ser de ayuda para el análisis del caso chileno. Al respecto, hemos querido enfatizar los principales problemas en el análisis de impactos -la complejidad de las aproximaciones en la literatura y lo difuso de las evidencias disponibles-, de forma que sean considerados en posteriores estudios sobre el tema.

En principio, creemos que el sistema chileno de AC y acreditación se encuentra aún en un estadio de ajustes y cambios, por lo que sus impactos resultan todavía muy difíciles de anticipar. La Ley 20.129 estableció bases institucionales y arquitectura consensuada para la proyección del sistema; sin embargo, tendrá que pasar más tiempo para establecer un juicio sobre la dirección que éste adoptará en Chile, cómo se consolidarán los distintos roles asignados y, por cierto, cuáles podrían llegar a ser sus impactos posibles. Con todo, queremos ofrecer aquí ciertas consideraciones indispensables en el análisis de los impactos del AC y, a la vez, aventurar algunas pocas proposiciones -ciertamente muy generales- que contribuyan en la problematización y estudio futuro del tema.

\section{Problemas para el estudio de los impactos de los procesos de AC y acreditación}

Las políticas de AC han logrado ocupar un lugar central en el contexto de la educación superior en Chile y el resto el mundo. Aun cuando se trata de políticas relativamente recientes, existe cierto consenso de que son prácticas que se han extendido ampliamente y han llegado para quedarse. Producto del desarrollo de dichas políticas -principalmente en Europa occidental y EE.UU.-, desde la década de los noventa emerge un debate orientado a identificar sus principales impactos y resultados, encontrándose con diversas dificultades de carácter metodológico y conceptual. 
Un primer aspecto problemático se relaciona con el concepto de "calidad" inmerso en el AC, con múltiples aproximaciones en la literatura, no existiendo acuerdos generalizados sobre su sentido último. La calidad en educación superior permanece como un concepto amplio y relativo, interpretado de forma diferente según el contexto y la perspectiva desde la cual se le observa (Harvey $\&$ Green, 1993). Por ello, a la hora de evaluar el impacto del AC hay que tener presente que los distintos actores entienden calidad de un modo diferente, por lo que no resulta sencillo-ni conveniente-fijar de modo estricto y único la dirección ni magnitud del cambio de calidad esperado. En Chile, la ley que establece el sistema de acreditación no contempla una definición precisa de la calidad, más bien se aproxima a ella de un modo amplio y flexible.

Una segunda complicación se refiere a la consideración del contexto en que se diseñan e implementan las políticas de AC y los procedimientos de acreditación, puesto que no se puede suponer neutralidad, sino más bien circunstancias muchas veces cargadas de contenido político (Englert, 1986). Lo anterior tiene consecuencias prácticas, porque los propósitos y el modo mediante el cual se desarrolla el AC se desprenden y se dan en dicho contexto. Así, es posible encontrar casos en los que cierto discurso (sobre mejoramiento, por ejemplo) puede esconder otros intereses y presiones (Harvey $\&$ Newton, 2004), tanto por parte de quienes conducen la evaluación como por aquellos que son objeto de ella. La mirada sobre el impacto se enreda cuando la política se da en un contexto en el que participan diversos actores con motivaciones diferentes -algunas explícitas, otras implícitas-, existiendo intereses variados que pueden ir desde el genuino interés por el mejoramiento o el direccionamiento del sistema hacia una mayor transparencia, hasta el solo propósito de obtener una certificación pública en contextos de mercado o bien limitar lo que para algunos puede ser una excesiva diversidad.

En tercer lugar está la evidente dificultad para aislar las causas y efectos, pues los instrumentos de AC son sólo uno de los varios factores que pueden influir en el desarrollo de las instituciones de educación superior (Stensaker, 2003). Ciertamente, esto ocurre en el caso chileno, puesto que el AC ha sido acompañado con otras políticas 
$y$ en un contexto de una variedad de iniciativas que coinciden en el tiempo (Allard, 2000).

\section{Dinámicas comprometidas y respuestas variadas}

Es indispensable reconocer que el aseguramiento externo de la calidad y en particular la acreditación deben analizarse en relación con las prácticas internas de AC de las propias instituciones (Askling, 1997). En general, se entiende que son los procesos internos de AC los que redundan en un impacto más profundo y permanente en el mejoramiento de la calidad. Los procesos externos de AC son muchas veces necesarios, pero no suficientes. Por su parte, algunos de estos procesos externos pueden presentar, en ocasiones, sesgos marcados al control y rendición de cuentas (accountability), en desmedro y contraposición al mejoramiento (Trow, 1996).

Que las políticas externas de AC lleven a procesos internos de AC y que estos últimos, a su vez, conduzcan a cambios e impactos sobre la calidad de la educación superior es un argumento complejo y difícil de probar desde el punto de vista metodológico. Con todo, la apuesta es justamente esa: promover cambios de la calidad, de un modo indirecto.

En el AC y los procesos de acreditación se dan dinámicas complejas en las que participan, al menos, una agencia y sus evaluadores y las instituciones y sus diversos actores involucrados.

La acción externa no puede verse aisladamente de lo que puede pasar internamente. Existen propósitos, motivaciones e intereses comprometidos en uno y otro lado. En un estudio a propósito de la evolución del sistema de acreditación en Estados Unidos, Peter Ewell (2007) analizó cómo las instituciones pueden responder de diverso modo a los distintos énfasis de los procesos externos de AC, ilustrando la relación como si fuera un juego en el que las instituciones van ajustando permanentemente sus respuestas a las exigencias, igualmente cambiantes, de las agencias. Así, es esperable que un proceso enfocado a los insumos y procesos, por ejemplo, obtenga de las instituciones respuestas diferentes que otro orientado 
a la evaluación de resultados, como pareciera ser -al menos en los dichos- la evolución del proceso de acreditación en Chile.

A lo anterior habría que agregar algo que complica aún más la relación entre las dimensiones externa e interna de AC, y es que no parece sensato que todas las instituciones respondan de idéntico modo a los procesos de acreditación. Frente a un idéntico procedimiento de evaluación/acreditación puede ocurrir, por ejemplo, que ciertas instituciones respondan de manera fundamentalmente reactiva, ajustando su actividad a las exigencias de la agencia, mientras que otras generen importantes niveles de resistencia frente a una intervención que es vista como ajena a los núcleos académicos internos, o bien que algunas establezcan un amplio espacio a la colaboración y asuman los procesos de acreditación como un instrumento de mejoramiento significativo.

Por cierto que es parte de las normas del juego que las instituciones respondan del modo más apropiado a los requerimientos planteados, haciendo uso de su autonomía y especificidad propia. Sin embargo, puede darse el caso de que instituciones de distinta naturaleza, no sólo en términos de su misión y cultura, sino que fundamentalmente por las motivaciones y desafíos que enfrentan en contextos de fuerte competencia, respondan de modo significativamente distinto a los procedimientos y criterios de la acreditación.

Jethro Newton (2000), a partir de un estudio de caso de percepciones de los académicos respecto del aseguramiento y monitoreo de la calidad, sugiere que las respuestas que pueden darse en cuanto a los procesos externos son variadas, desde aquellos que fundamentalmente responden de un modo ritual y reactivo (feeding the beast), hasta aquellos que efectivamente asumen de modo honesto el objetivo del mejoramiento de la calidad. En el primer caso se advierte del riesgo de burocratización de los procesos de AC y acreditación, en la medida en que son muy poco efectivos con ciertas instituciones (Barrow, 1999). Frente a las instituciones inmunes y reacias a procedimientos de promoción de la calidad, muchas veces sólo queda un enfoque básico de control y supervisión. 
El análisis de impacto de los procesos de AC y acreditación presenta diversas dificultades conceptuales y metodológicas, a la vez que se da en contextos de relaciones muy complejas. Frente a esto, resulta recomendable aproximarse mediante una perspectiva amplia y suave (Stensaker, 2007), que permita ponderar las diversas consideraciones y mantener cautela frente a la complejidad del asunto. La mirada sobre los impactos no debe limitarse a la medición y dimensionamiento objetivo de ciertos cambios; debe más bien atender a los diferentes procesos que se desarrollan de forma interrelacionada y que pueden dar cuenta del curso de acción de una política y de los actores en ella involucrados (Cerych, 1984).

\section{Los impactos desde la perspectiva de los propósitos}

Si bien la implementación de toda política lleva consigo efectos que no obedecen estrictamente a su finalidad declarada, la pregunta acerca del grado de cumplimiento de los objetivos inicialmente planteados es siempre útil. Sin embargo, nuevamente, no se trata de una pregunta sencilla, puesto que los propósitos que persiguen el AC y la acreditación pueden variar según el contexto y las instituciones que participan.

La preocupación actual por la calidad parece responder principalmente a algunas tendencias de carácter más o menos global, lo que explica la gran cantidad de países que han implementado políticas de AC en la educación superior. Se trata, principalmente, de los desafíos que implica la transición de una educación superior de elite a una de masas, así como del giro que ha experimentado la concepción de la gestión y regulación pública (OECD, 2008). Billing ha estudiado cómo los distintos países han establecido los propósitos de sus sistemas de AC y cómo la literatura e investigación ha intentado identificar categorías, tendencias y sus principales convergencias. Sin embargo, las diferencias constatadas parecen ser tanto o más importantes que las similitudes. Respecto de las principales semejanzas, los siguientes objetivos son los más usuales en los sistemas nacionales de AC (Neave, 1991; Kells, 1999; Van Vught \& Westerheijden, 1994; Vroeijenstijn, 1995; Billing, 2004), aun cuando en su implementación pueden ser entendidos de modos 
muy distintos: Fomento y mejoramiento de la calidad; Control y accountability; Información pública y sobre estándares de calidad; Certificación-acreditación; Apoyo a la planificación institucional y del sistema, y otros, tales como requisitos/direccionamiento del financiamiento, reconocimiento y rankings.

De los anteriores, el modelo chileno ha hecho mención a su mayoría, aun cuando, en un análisis más detallado, puede plantearse que existen ciertos acentos hacia el fomento de la calidad, el control, la acreditación y la información pública. Los dos primeros son los que han generado mayor controversia en el debate internacional, que discute acerca de la permanente tensión entre las funciones de "mejoramiento" y "accountability" (Westerheijden, D. F., Stensaker, B. \& Rosa, M., 2007). Sobre esto, algunos han sostenido que existiría cierta incompatibilidad entre ambos objetivos, por lo que resultaría imposible abordarlos al mismo tiempo. Sin embargo, en la práctica se observa que los esquemas de AC consultan estos objetivos simultáneamente, incluso siendo difícil distinguir si existe cierto énfasis en alguno de ellos en particular, ya que se ha buscado explícitamente un equilibrio que permita cumplir objetivos de mejoramiento y accountability simultáneamente (Maassen, 1998; Dano $\&$ Stensaker, 2007). En este sentido, se ha señalado que mejoramiento y "accountability" son objetivos muy estrechamente relacionados entre sí, lo que hace inviable su separación (Proitz et al., 2004).

El sistema chileno de AC también ejemplifica la búsqueda de un equilibrio, combinando diversos objetivos simultáneamente a través de sus procesos. Mientras el licenciamiento enfatiza en el control, los procesos de acreditación ponen el acento en el mejoramiento continuo.

Los procesos de licenciamiento en Chile, restringidos fundamentalmente a las instituciones privadas, buscaron introducir prácticas orientadas a fomentar y establecer capacidades de autorregulación en las instituciones, a la vez de garantizar un nivel mínimo satisfactorio de desarrollo de los proyectos institucionales. 
Sin embargo:

"La falta de consistencia entre el proceso de licenciamiento y su efecto final representa un problema: todo el proceso apunta a la evaluación de un proyecto institucional y su implementación. La definición de autonomía, en cambio, implica precisamente la posibilidad de modificar radicalmente el proyecto que condujo a ella y el proceso -tal como está definido en la LOCE- no tiene mecanismos que permitan resolver esta contradicción" (Lemaitre, 2005).

Mientras que algunas instituciones privadas que alcanzaron su plena autonomía continuaron en un proceso de desarrollo permanente, otras, en ausencia de regulación externa, modificaron sustancialmente sus proyectos originales, optaron por crecimientos en extremo inorgánicos o bien, simplemente, cambiaron de propiedad iniciándose como proyectos nuevos.

El modelo de acreditación institucional, por su parte, se ha planteado como una auditoría académica, con un tipo de aproximación y criterios que presentan un claro acento hacia la definición e implementación de una serie de políticas y mecanismos de AC en el desarrollo de la gestión institucional y la docencia de pregrado impartida. En este sentido, se trata de un modelo que busca impactar en el mejoramiento continuo de las instituciones a través del robustecimiento del aseguramiento interno, pero que, sin embargo, exige una línea básica mínima de calidad y capacidades instaladas de autorregulación.

La acreditación institucional ha tenido que hacerse cargo de una variedad de instituciones, algunas de larga trayectoria y otras nuevas, algunas con capacidades previas de autorregulación y otras no necesariamente. De este modo, la acreditación, aun cuando se propone el mejoramiento continuo, en ocasiones ha tenido que verificar y garantizar una línea mínima en el cumplimiento de ciertos criterios de evaluación en instituciones que se han incorporado al proceso con muy bajos niveles de calidad, incluso para su permanencia dentro del sistema. 
En consecuencia, el AC y la acreditación en particular -que busca impactar por cierto en su propósito de mejoramiento de la calidad- han necesitado a la vez enfrentar con un mismo procedimiento a instituciones que se encuentran en muy distintas situaciones de entrada, algunas de ellas muy precarias.

Entonces, desde el punto de vista del impacto del AC y la acreditación, es particularmente complejo el análisis del cumplimiento de propósitos, puesto que se superponen y experimentan de modo distinto según las instituciones que participan.

Adicionalmente, la acreditación tiene el propósito de contribuir con más y mejor información en el sistema de educación superior, el que regulado por sí solo es susceptible de presentar múltiples asimetrías de información (Dill \& Soo, 2004). En Chile parece que los resultados de los procesos de acreditación han concitado fuerte interés en la opinión pública. Las agencias responsables de conducir la acreditación se han preocupado de divulgar sus juicios finales y muchas instituciones hacen uso publicitario de ellos.

El hecho de que los resultados de la acreditación puedan efectivamente impactar en el propósito de entregar más y mejor información al público es, sin embargo, todavía incierto. En efecto, el impacto de la acreditación en la percepción de los estudiantes y público en general es una cuestión todavía no estudiada (Westerheijden, Hulpiau \& Waeytens, 2007) y que, a nuestro juicio, se encuentra relacionada con muchas otras variables y en un contexto donde imperan las dinámicas de mercado. Por de pronto, el AC -si se lo mira en detalle en su evolución y potencialidades- ha sido diseñado para acompañar a las fuerzas del mercado en la coordinación del sistema, no para sustituirlas (Brunner, 2008). De tal forma, los impactos o resultados esperables de los procesos de AC en materia de información al menos no son ajenos a lo que las dinámicas de mercado permiten.

Finalmente, la diversidad de propósitos que considera el AC y la acreditación plantea múltiples inconvenientes para la evaluación de sus impactos. Se trata de propósitos que se encuentran con contextos e 
instituciones diversas y que por lo mismo harán más o menos sentido según sea el caso.

\section{Algunas evidencias de la literatura}

Se ha planteado que existen tres tipos de análisis sobre el impacto del AC: i) los de aproximación teórica o de opinión, que tienden a predecir los posibles efectos de evaluación y acreditación; ii) los que se basan en evidencia limitada -mucha de ella anecdótica- y percepciones de los actores, y iii) los elaborados a partir de una recolección sistemática de datos (Harvey, 1999). La mayor parte de la literatura sobre impacto pareciera corresponder a los dos primeros tipos de análisis. En Chile, los estudios más significativos han sido desarrollados y recogidos por el Centro Interuniversitario de Desarrollo (CINDA) y por el Consejo Superior de Educación (CSE), existiendo una variedad de aproximaciones conceptuales, de análisis de casos y descripción de experiencias.

De la literatura local, uno de los impactos que parecen más recurrentes se vincula con los cambios en la cultura de las instituciones (Silva, 2006; Lemaitre, 2005; González, 2008). El AC y la acreditación han instalado en el centro del debate el tema por la calidad y una cierta lógica de razonamiento y prácticas de evaluación que no se encontraban del todo desarrolladas al interior de las instituciones. Asimismo, se perciben cambios significativos en el ámbito organizacional, en cuanto a la formalización de la dirección y orientaciones estratégicas (planes de desarrollo), una mayor apertura a la participación académica y diálogo sobre calidad, generación de información para la toma de decisiones y diversos ajustes, y una tendencia hacia la profesionalización de la gestión institucional y de la docencia impartida (Silva, 2005; Sepúlveda, 2005; Peña, 2005; Lemaitre, 2005; Mora, 2006; González, 2008).

Un estudio observa cómo se dan ciertas innovaciones educativas a propósito de la confluencia de las políticas de acreditación y las de financiamiento del Programa de Mejoramiento de la Calidad y Equidad de la Educación Superior (MECESUP), resaltando innovaciones particularmente en los temáticas de gestión curricular y procesos 
educativos, y de recursos y soportes del aprendizaje (Canales, De los Ríos y Letelier, 2008).

Con todo, pese a que el AC y la acreditación son percibidos como una de las transformaciones más importantes en nuestra educación superior desde la reforma de 1980 (Brunner, 2007), se considera aún como un sistema en construcción, que es preciso acompañar y ajustar en sus transformaciones más significativas (Navarro, 2007), y cuyos resultados más permanentes sólo podrán ser observados en el largo plazo (Mora, 2006).

En el ámbito internacional existe amplia literatura e investigaciones que confirman, en parte, aquellos temas observados por los estudios nacionales. Como hemos sostenido, se aprecian sin embargo diversas controversias sobre la dificultad de acceder a la dimensión de los impactos. Stensaker (2008) sintetiza los resultados de distintos estudios realizados internacionalmente identificando cuatro ámbitos de cambios recurrentes: i) distribución y ejercicio del poder al interior de las instituciones; ii) gestión organizacional y su profesionalización; iii) imagen que proyectan las instituciones al exterior (relaciones públicas), y iv) cierta permeabilidad y generación de más y mejor información para la toma de decisiones.

Sin embargo, los diversos estudios sobre impactos del AC y la acreditación mantienen serias dificultades para proveer evidencia sustancial con relación a lo que resulta más importante, esto es, el impacto en la calidad del proceso de enseñanza-aprendizaje ${ }^{4}$ (Stensaker, 2008). En cambio, se han privilegiado aquellos estudios que intentan describir los cambios ocurridos tras los procesos de evaluación en el plano organizacional (Brennan \& Shah, 2000) o bien el impacto percibido por los diversos actores institucionales (Newton, 2002; Horsburgh, 1999; Bornman et al., 2006).

4 Una excepción son los estudios derivados de la evaluación de los cambios de la ABET, agencia de acreditación americana en el área de ingeniería. Sus resultados son particularmente interesantes, constatando cambios significativos en la educación impartida a partir de las percepciones de estudiantes, académicos, empleadores y directivos (Terenzini et al., 2006). 
Las dificultades en la evaluación de impactos se pueden apreciar en la gran mayoría de los estudios. No parece haberse encontrado aún un método suficientemente robusto como para identificar si los cambios generados por el aseguramiento de la calidad se han traducido efectivamente en mejoras en la enseñanza-aprendizaje. Por otro lado, tampoco se puede caer en una postura naïve y asumir que las acciones implementadas en respuesta a las recomendaciones externas conducen inmediatamente a la superación de los problemas académicos de las universidades (Harvey, 2006).

\section{Finalmente}

Como síntesis, sugerimos las siguientes consideraciones y proposiciones que pueden ser útiles para el análisis de impactos en estudios futuros:

Existen enormes dificultades para el estudio de los impactos de las políticas de AC. Sin embargo, es sensato sostener que estos procesos no pasan desapercibidos y conllevan cambios posibles de ser anticipados y, eventualmente, otros no previstos.

Es conveniente asumir una aproximación amplia y abierta que considere que el AC no es una variable autónoma, sino que va acompañada con otros instrumentos de políticas y las dinámicas propias del sistema de educación superior.

El AC tiene una dimensión externa y otra interna a las instituciones, cuyas dinámicas no son estables ni necesariamente permanentes en el tiempo. Más bien, la evolución en las reglas del juego y el ajuste de la política entre las agencias e instituciones forman parte de la naturaleza misma del AC.

Igualmente, aun cuando es indispensable estudiar los propósitos del AC, estos varían en el tiempo, ajustándose a los cambios en el sistema de educación superior y la realidad de las instituciones.

No es posible sostener que todas las instituciones tienen las mismas motivaciones y circunstancias, o que acceden a los procesos 
de acreditación en idénticas condiciones. Por ello, la naturaleza de los procesos y su impacto son razonablemente diferenciados según el contexto y tipo de institución que se trate.

Pese a los problemas metodológicos y conceptuales, existen ciertos consensos sobre el impacto del AC en ciertas áreas temáticas. Sin embargo, no es claro cuán profundos y permanentes sean estos cambios, lo que dependerá en gran medida de la evolución del sistema.

Una preocupación especial se requiere en el análisis de estos procesos respecto de las mejoras en la enseñanza-aprendizaje, lo que constituye, a fin de cuentas, uno de los aspectos centrales en los que se espera generar cambios significativos.

Para concluir, no quisiéramos ignorar que probablemente lo más valioso, desde las perspectivas del impacto, es precisamente el desarrollo de procesos genuinos de evaluación al interior de las instituciones. En este sentido, lo central está en el proceso mismo y la manera mediante la cual las instituciones hacen uso de un instrumento que, con todas sus limitaciones, sirve de vehículo para llevar a cabo las transformaciones necesarias para el mejoramiento continuo en el contexto propio de una institución.

\section{Referencias bibliográficas}

Allard, R. (2000) Políticas Públicas en Educación Superior en Chile: Contexto, Programas y Proyección. Revista de la Educación Superior Chilena. MECESUP. Santiago de Chile.

Armanet, P. (2005) Acreditación de la Calidad en Chile 1999-2005. Santiago de Chile: Serie Seminarios Internacionales, Consejo Superior de Educación.

Askling, B. (1997) Quality monitoring as an institutional enterprise. Quality in Higher Education, 3.

Barrow, M. (1999) Quality management systems and dramaturgical compliance. Quality in Higher Education, 5(1).

Billing, D. (2004) International comparisons and trends in quality assurance of higher education: commonality or diversity? Higher Education, 47. 
Bornmann, L.; Mittag, S. \& Danie, H.D. (2006) Quality assurance in higher education meta-evaluation of multi-stage evaluation procedures in Germany. Higher Education, 52(4), pp. 687-709.

Brennan, J. and Shah T. (2000) Quality Assessment and Institutional Change: Experiences from 14 Countries. Higher Education, 40.

Brunner, J.J. \& Uribe, D. (2007) Mercados Universitarios, el nuevo escenario de la educación superior. Santiago de Chile: Universidad Diego Portales.

Brunner, J.J. (2008) Educación Superior en Chile: Instituciones, Mercados y Politicas Gubernamentales, 1967-2007. Tesis doctoral.

Canales, De los Ríos y Letelier (2008) Comprendiendo la Implementación de Innovaciones Educativas Derivadas de Programas MECESUP y CNAP para Ciencia y Tecnología. Santiago de Chile: CICES-Universidad de Santiago de Chile.

Cerych, L. (1984) The Policy Perspective. En: Clark, B. (Ed.) Perspectives on Higher Education. University of California Press.

Dano, T. \& Stensaker, B. (2007) Still Balancing Improvement and Accountability? Developments in External Quality Assurance in the Nordic Countries 1996-2006. Quality in Higher Education, 13(1).

Dill, D. D. \& Soo, M. (2004) Transparency and Quality in Higher Education Markets. En: Teixeira, P.; Jongbloed, B.; Dill, D. and Amaral A. (Eds.) Markets in Higher Education: Rhetoric or Reality? Dordrecht, the Netherlands: Kluwer, pp. 61-86.

Englert, Richard (1986) Higher Education's Evaluation Politics. En: Grove $\&$ Stauffer (Eds.) Policy Controversies in Higher Education. New York: Greenwood Press.

Ewell, Peter (2007) The quality game: external review and institutional reactions over three decades in the US. En: Westerheijden et al. (eds.) Quality Assurance in Higher Education: Trends in regulation and transformation. Higher Education Dynamics 20.

González, E. (2005) Perfiles de egreso y procesos de acreditación. En: Aseguramiento de la calidad: impacto y proyecciones. Serie Seminarios Internacionales. Santiago de Chile: Consejo Superior de Educación.

González, L.E. (2008) El impacto del proceso de evaluación y acreditación en las universidades de América Latina. CINDA-IESALC/UNESCO.

Harvey \& Green (1993) Defining quality. Assessment \& Evaluation in Higher Education, 18(1).

Harvey \& Newton (2004) Transforming quality evaluation. Quality in Higher Education, 10(2). 
Harvey, L. (1999) Evaluating the evaluators. Presentación realizada en Fifth Biennial Conference, International Network of Quality Assurance Agencies in Higher Education, Santiago de Chile.

Harvey, L. (2006) Impact of Quality Assurance: Overview of a discussion between representatives of external quality assurance agencies. Quality in Higher Education, 12(3).

Horsburgh, M. (1999) Quality monitoring in higher education: the impact on student learning. Quality in Higher Education, 5(1).

INQAAHE Review Panel (2005) Report of an Evaluation of the Comisión Nacional de Acreditación de Pregrado, CNAP. Team of international experts of the International Network for Quality Assurance Agencies for Higher Education, INQAAHE. December.

Kells, H. (1999) National Higher Education Evaluation Systems: Methods for analysis and some propositions for the research and policy void. Higher Education, 38.

Lemaitre M.J. (2005) Aseguramiento de la calidad en Chile: impacto y proyecciones. En Aseguramiento de la calidad: impacto y proyecciones. Santiago de Chile: Serie Seminarios Internacionales, Consejo Superior de Educación.

Levy, Daniel (2002) Latin America's Tertiary Education: Accelerating Pluralism. Paper presented at the seminar Higher Education and Science Technology in Latin America and the Caribbean: Responding to Expansion and Diversification. Fortaleza, Brazil.

Libertad y Desarrollo (2003) Exceso de Oferta de Profesionales: un Mito. Temas Públicos, 612.

Libertad y Desarrollo (2003b) Calidad en Educación Superior: ¿Conviene la Acreditación? Temas Públicos, 632.

Maassen, P. (1998) Quality Assurance in the Netherlands. New Directions for Institutional Research, 25(3).

Mora, A. (2006) Impacto de los procesos de acreditación en la Universidad de Concepción. Calidad en la Educación, 24.

Navarro, G. (2007) Impacto del proceso de acreditación de carreras en el mejoramiento de la gestión académica. Calidad en la Educación, 27.

Neave, G. (2001) Educación Superior: Historia y Política. Barcelona: Gedisa.

Newton, J. (2000) Feeding the beast or improving quality? Academic's perceptions of quality assurance and quality monitoring. Quality in Higher Education, 6(2). 
Newton, J. (2002) Views from below: academics coping with quality. Quality in higher education, 8(2).

OECD (2008) Tertiary Education for the Knowledge Society. Thematic Review of Tertiary Education. Organisation for Economic Co-Operation and Development.

Peña, C. (2005) Los procesos de acreditación en la Universidad Diego Portales. En Aseguramiento de la calidad: impacto y proyecciones. Santiago de Chile: Serie Seminarios Internacionales, Consejo Superior de Educación.

Proitz T.S.; Stensaker B. \& Harvey L. (2004) Accreditation, standards and diversity: an analysis of EQUIS accreditation reports. Assessment $E$ Evaluation in Higher Education, 29(6).

Sepúlveda, C. (2005) El proceso de acreditación institucional y propuestas de mejoramiento de la Universidad de Chile. En Aseguramiento de la calidad: impacto y proyecciones. Santiago de Chile: Serie Seminarios Internacionales, Consejo Superior de Educación.

Silva, C. (2005) Experiencia del Instituto Profesional DuocUC en la acreditación institucional. En Aseguramiento de la calidad: impacto y proyecciones. Serie Seminarios Internacionales, Consejo Superior de Educación.

Silva, C. (2006) Aseguramiento de calidad en gestión de instituciones de educación superior: expectativas y desafíos. Calidad en la Educación, 24.

Stensaker, B. (2003) Trance, transparency and transformation: the impact of external quality monitoring in higher education. Quality in Higher Education, 9.

Stensaker, B. (2007) Impact of Quality Processes. En: Embedding Quality Culture in Higher Education. A Selection of Papers from the First European Forum for Quality Assurance. München.

Stensaker, B. (2008) Outcomes of Quality Assurance: A Discussion of Knowledge, Methodology and Validity. Quality in Higher Education, $14: 1$

Trow, Martin (1996) On accountability of higher education in the US. En: Bowen, W. \& Shapiro, H. (Eds.) Universities and their Leadership. Princeton: Princeton University Press.

Van Vught, F.A. \& Westerheijden, D.F. (1994) Towards a general model of quality assessment in higher education. Higher Education, 28(3).

Vroeijenstijn, A. (1995) Improvement and accountability, navigating between Scylla and Charybdis. Guide for quality assessment in higher education. London: Jessica Kingsley. 
Westerheijden, D. F; Stensaker, B. \& Rosa, M, (2007) Conclusions and further challenges. En Westerheijden, D. F, Stensaker, B. \& Rosa, M. (Eds.). Quality assurance in higher education. Dordrecht, The Netherlands: Springer.

Westerheijden, Hulpiau \& Waeytens (2007) From Design and Implementation to Impact of Quality Assurance: An Overview of Some Studies into what Impacts Improvement. Tertiary Education and Management, 13(4), pp. 1573-1936.

Recibido: 29 de septiembre de 2009

Aceptado: 23 de octubre de 2009 